\title{
Determination of Coil Inductances Cylindrical Iron Nucleus
}

\author{
Azeddine Mazouz ${ }^{1}$, Djemai Hidjazi ${ }^{2}$, Aziz Chaghi $^{3}$ \\ Universite de batna rue chahid boukhlouf batna 05000 \\ e-mail: doude05@yahoo.fr, d_hidjazi@yahoo.fr, az_chaghi@univ-batna.dz
}

\begin{abstract}
The coil iron nucleus cylindrical is a nonlinear electro radio in which the moving of the nucleus in a sense or in other causes change in inductance and can reach extreme values at the superposition of nucleus and coil centers. The variation of the inductance and the degree of freedom of movement of the nucleus can lead to a device with electromechanical conversion The aim of this paper is the determination and visualization of self inductance and mutual of the (C.I.C.C) based on geometric dimensions and the displacement of the nucleus.
\end{abstract}

Keywords: Coil, iron, nucleus cylindrical, linear motor, inductance

\section{Introduction}

The cylindrical coil with iron nucleus can be explored as a tubular linear motor oscillation (OTLM) [3]. This type of engine is based on the periodic variation of the inductance, which causes the change in the behavior of the electrical circuit with the existence of a series capacitance.

The OTLM with mobile induct exists in two disposition, vertical and horizontal [4], [5]. The stationary part consists of a solenoid which forms part of the resonant RLC circuit. The ferromagnetic cylinder inserted in the solenoid constitutes the moving part.

Parametric electromechanical elements, comprehend a class of single machine which present certain common characteristics [6], [7]. The process of energy conversion is similar to some type of asynchronous motor [1]. Power conversion is due to a regular change of the parameters, that is why is called parametric machines. The functioning of this type of machine is based on the application of electromechanical oscillations. [7] and [8].

\section{Inductances as Function of Core Displacement}

The ferromagnetic nucleus core is integral part which reflects the position or displacement. This nucleus is pushed to a variable length if in a coil of $N$ turns uniformly distributed over a length 'l' as shown by figure 2.1.

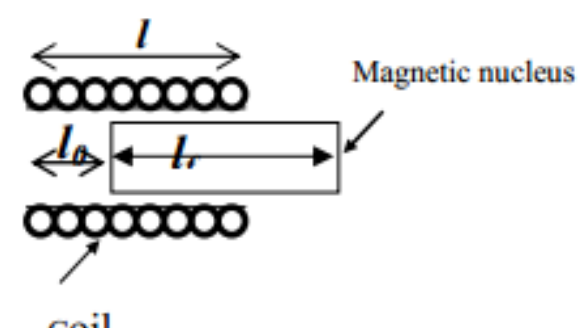

Figure 2.1 Diagram of a coil with plunger

The self-induction coil coefficient $L$ depends on the penetration of the nucleus. The calculation of $L$ is done by considering the inductor as the association of an inductance of the air length 10 , with a self-induction coefficient $L_{0}$ in series with an inductance iron nucleus of length If, with self-induction coefficient of $L_{f}$ and a mutual $M$. 
This inductance is expressed as:

$L=L_{0}+L_{f}+2 M$

With:

$$
M=K \sqrt{L_{0} \cdot L_{f}}
$$

$\mathbf{k}$ is the coupling coefficient which is assumed constant $(0 \leq k \leq 1)$ Depending on various characteristics of the circuit we can write:

$$
L_{0}=\mu_{0} \cdot \frac{N^{2}}{l^{2}} S_{0} \cdot l_{0}=\mu_{0} \cdot \frac{N^{2}}{l^{2}} S_{0}\left(l-l_{f}\right)
$$

Determination of Coil Inductances Cylindrical IronNucleus

$$
L_{f}=\mu_{0} \cdot \frac{N^{2}}{l^{2}}\left[S_{0}+\left(\mu_{f}-1\right) S_{f}\right] l_{f}
$$

The expression of $L$ is then:

$$
L=\mu_{0} \cdot \frac{N^{2}}{l^{2}}\left(S_{0} l+\left(\mu_{f}-1\right) S_{f} l_{f}+2 k \sqrt{S_{0}\left[S_{0}+\left(\mu_{p}-1\right) \cdot S_{f}\right]} \cdot \sqrt{\left(l-l_{f}\right)} \cdot l_{f}\right)
$$

The displacement $\Delta l_{f}$ of the nucleus, leads to a variation $\Delta L$ of the inductance, which depends on If which is a nonlinear function of $\Delta I_{f}$ as in the case of air gap variable inductance of. [2].

\section{Inductances Equations Development}

To determine the expression (1) we need to calculate the inductances $L_{0}, L_{f}$ and $M$ :

\subsection{Inductance without Nucleus}

In this case the expression of the inductance is given by:

$$
L_{0}=\mu_{0} \frac{N^{2}}{l} S_{0} \cdot \frac{l_{0}}{l}=\mu_{0} \frac{N^{2}}{l} \cdot \frac{S_{0}\left(1-l_{f}\right)}{l}
$$

Where: $I=I_{0}+I_{\mathrm{f}}$

$S_{0}$ is the vacuum section

$$
l_{0}=\mu_{0} \frac{N^{2}}{l} S_{0}-\frac{\left(\mu_{n} N^{2} l_{f}\right)}{l}
$$

We have:

$$
L_{\propto}=\mu_{0} \cdot \pi \cdot r^{2} \cdot n^{2} \cdot l
$$

With: $n=\frac{N}{l}$

So:

$$
\Longrightarrow L_{\propto}=\frac{\mu_{0} \cdot N^{2} \cdot S_{0}}{l^{2}} \cdot l=\frac{\mu_{0} \cdot N^{2} \cdot S_{0}}{l}
$$

$$
L_{\square}=L_{\infty}-L_{\infty} \frac{L_{f}}{l}=L_{\infty<}\left(1-\frac{L_{f}}{l}\right)
$$


If is a function of displacement $\mathrm{x}$, to be determined later.

We set: $a=L_{\infty}$

So:

$$
\begin{aligned}
b & =\frac{L_{\infty}}{l} \\
L_{0} & =a-b . h
\end{aligned}
$$

Inductance of the nucleus

$$
\left.L_{f}=\frac{\mathrm{H}_{c} \mathrm{~N}^{\mathrm{a}}}{v}\left[S_{0}+\left(\mu_{f}-1\right) \cdot S_{f}\right]\right]^{i f}
$$

With:

$$
\begin{aligned}
& L_{f}=\frac{L_{\infty}}{s_{0}}\left[s_{0}+\left(\mu_{f}-1\right) \cdot S_{f}\right] \cdot{ }_{i} \\
& L_{f}-\frac{L_{\infty}}{s_{n}}=l_{f}+L_{20} \cdot s_{f m}\left(\mu_{f}-1\right) \cdot l_{f}
\end{aligned}
$$

We set:

$$
\tau=h \cdot s_{s_{f}}^{s_{f}} \cdot\left(\mu_{f}-1\right)
$$

And we replace by the constant (b) we have:

$$
L_{z}=(b+c) \cdot 4
$$

\subsection{Mutual inductance}

In equation (2) we replace the expressions (10) and (14) we will have:

$$
M=\frac{L_{\infty}}{s_{0}} \frac{k}{l} \cdot \sqrt{S_{0}\left[S_{0}+\left(\mu_{f}-1\right) \cdot s_{f}\right] \cdot\left(l-l_{f}\right) \cdot l_{f}}
$$

We set: $d=S_{0}\left[S_{0}+\left(\mu_{f}-1\right) . S_{f}\right]$

And we replace the constant (b) we have:

$$
M-\frac{k}{s_{0}} \cdot b \cdot \sqrt{d \cdot\left(l-l_{f}\right) \cdot l_{f}}
$$

\subsection{Total Inductance (coil over nucleus)}

The total inductance of the coil is determined by taking the expressions of the different inductances obtained before

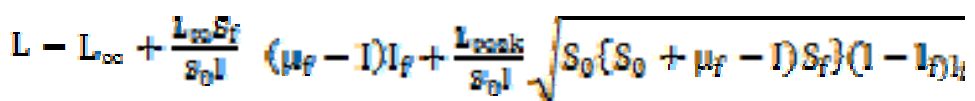

The inductance expressed as a function of the constants $(a, b, c$ and d) is:

$$
L=a+c_{n} l_{f}+\frac{2 \cdot k}{s_{n}} \cdot b \cdot \sqrt{d \cdot\left(l-l_{f}\right) \cdot l_{l}}
$$




\section{Coil Parameters}

\subsection{Coil without Iron Nucleus}

Under variable voltage of $50 \mathrm{~Hz}$ frequency, the parameters indicated in table 4.1 are measured by considering the circuit indicated in figure 4.1

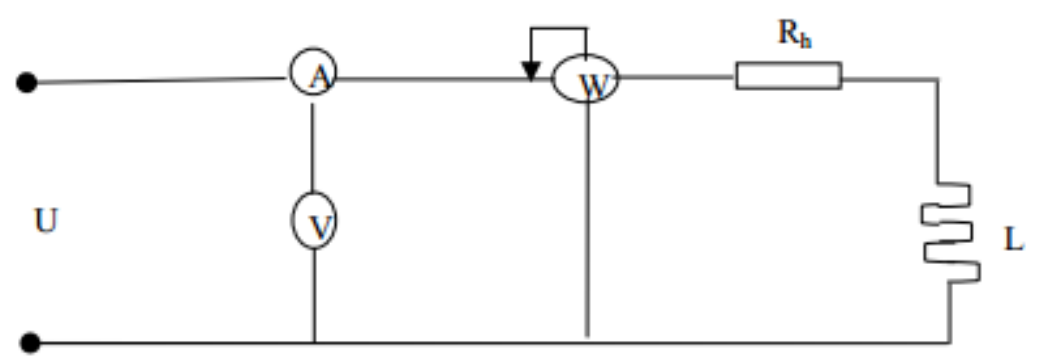

Figure 4.1 Practical measuring circuit

Table 4.1. Measured parameters

\begin{tabular}{ccc}
\hline UL $(v)$ & $\mathrm{I}(\mathrm{A})$ & $\mathrm{Z}$ \\
\hline 9.5 & 0.45 & 21.1 \\
13.0 & 0.6 & 22 \\
15.6 & 0.7 & 22.28 \\
18.3 & 0.8 & 22.87 \\
\hline
\end{tabular}

The inductive reactance: $X_{0}=\sqrt{Z^{2}-R^{2}}=L . \omega$;

Inductance: $\quad \mathrm{L}_{0}=\mathrm{X}_{0} / \omega$;

The impedance: $Z_{0}$ average $=22 \Omega$;

The internal resistance of the coil: $R=10.55 \Omega$;

The inductance of the coil without its nucleus $L_{0}=0.06 \mathrm{H}$.

The internal resistance of the coil without nucleus can be determined by using the same circuit feed by direct current.

\subsection{Coil with Iron's Nucleus}

Before performing the test, we must provide the core inside the coil and make sure it is centered and gradually feeding the circuit with an alternative voltage of $50 \mathrm{~Hz}$ frequency. The indications of measuring devices are shown in table 4.2

Table 4.2. The indications of measuring

\begin{tabular}{ccccccc}
\hline $\mathrm{UL}(\mathrm{v})$ & $\mathrm{I}(\mathrm{A})$ & $\mathrm{P}(\mathrm{w})$ & $\mathrm{Ir}$ & $\mathrm{Ur}$ & $\mathrm{UL}$ & $\mathrm{Z}$ \\
\hline 16,7 & 0.05 & 0.83 & 0.0025 & 332 & - & 334 \\
35 & 0.1 & 0.95 & 0.01 & 95 & - & 350 \\
53 & 0.15 & 1.05 & 0.0225 & 46.67 & 25.11 & 353.33 \\
96 & 0.3 & 2.06 & 0.09 & 22.89 & 93.23 & 320 \\
150 & 0.48 & 4.32 & 0.2304 & 18.75 & 148.82 & 312.5 \\
225 & 0.7 & 9.45 & 0.49 & 19.3 & 224.17 & 321.43 \\
\hline
\end{tabular}

with: Zaverage $=332 \Omega, \mathrm{R}=10,55 \Omega \quad, \mathrm{L}=1.116 \mathrm{H}$ 


\subsection{Inductance Variation in Function of Nucleus Position}

The coil with iron's nucleus is characterized by the variation of its inductance in function of the movement of the nucleus inside the coil. Taking reference as the superposition of the coil's and the nucleus's centre as indicated in figure 4.2.

The manually movement of the nucleus along the axis $X$ passes through 03 zones. For the left and right areas, the coil is without nucleus and the inductance takes a minimum value.

However for the intermediate area the nucleus occupied gradually the total of the coil to pursue the air that it contains and in this case the inductance becomes maximum.

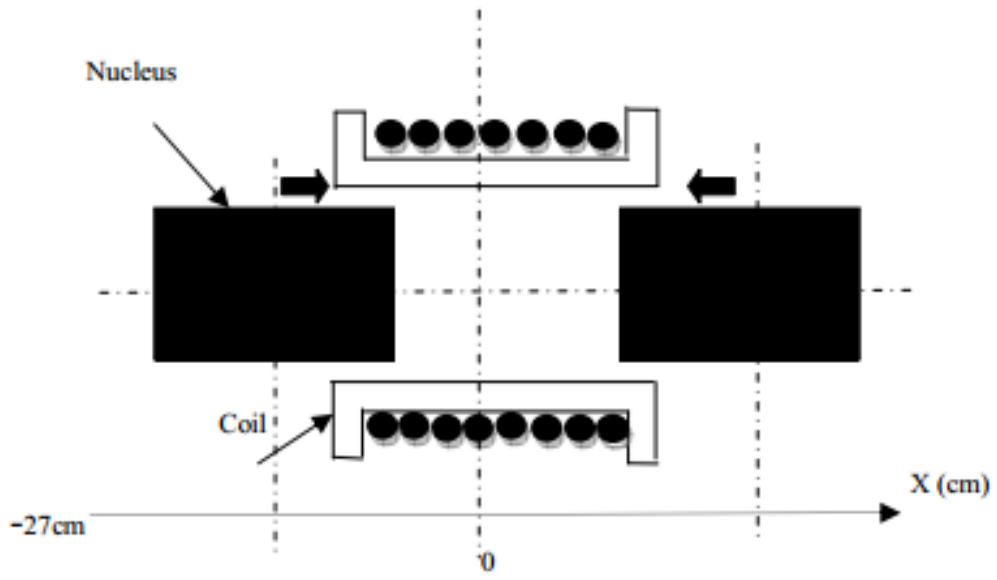

Figure 4.2. Representative patterns of the core displacement

The experiment results taken under a constant voltage of Ueff $=50 \mathrm{~V}$ with the assumption that the resistance stay constant, are shown by figure 4.3 .

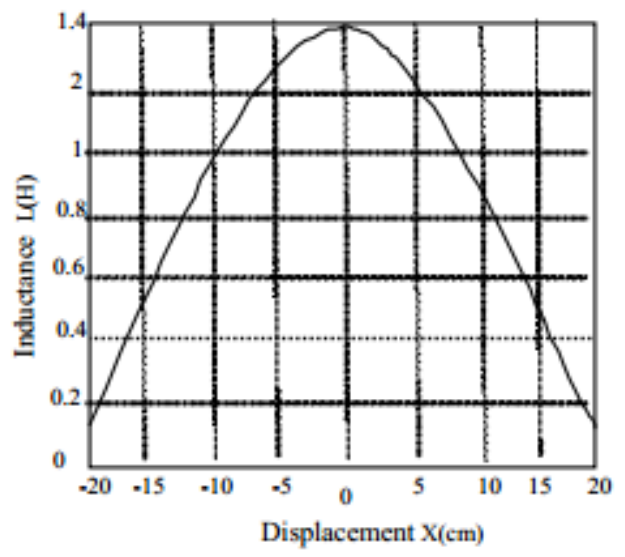

Figure 4.3. Variation of the inductance I in function of displacement $X$

\subsection{Determination of the Length of the Iron as Function of the Displacement}

The expressions [(11), (15), (17), (18)], of this article are function of length 10 and If inside the coil which are also function of $x$

The superposition of the two curves If $=f(x)$ and $I 0=g(x)$ presented in fig IV.4 shows the evolution of the gradual penetration of the nucleus into the coil by chasing the air in it. 


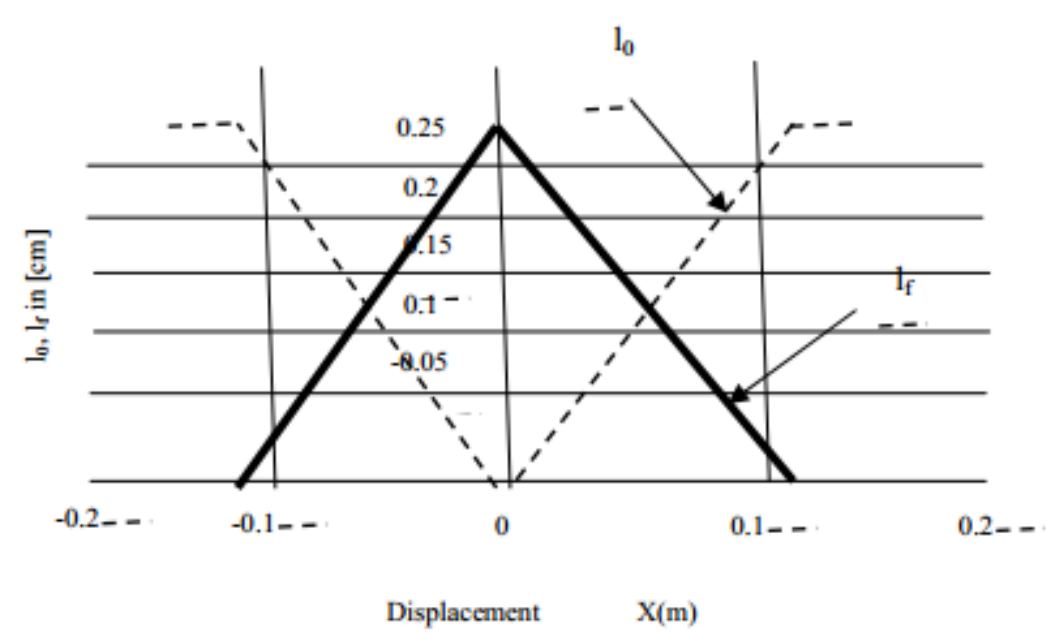

Figure 4.4. Superposition of curves 10 If giving:

The mathematics expressions of these two functions are determined by interpolation

$$
b_{f}=0.2 e^{\left(-200 x^{2}\right)}
$$

And

$$
l_{0}=1-\left(0.2 e^{\left(-200 x^{2}\right)}\right)
$$

From equations governing the behavior of the coil with and without a nucleus, a MATLAB program was developed for the calculation of inductances $L 0$, $L f$ and $M$, according to the dimensions of the coil and core taking into account the varying displacement of the core in the coil.

\section{Inductance of the Coil with Iron's Nucleus (Nucleus Center)}

An inductor with iron nucleus consists of a coil with 3000 spires and a length I with negligible resistance.

The core is composed of sheets of very good quality with a coefficient equal to 0.9 and with a length $\mathrm{In}$ as indicated in table 5.1

The coil is feed with a voltage of $115 \mathrm{~V}$ and a frequency $\mathrm{f}=50 \mathrm{~Hz}$.It is assumed that the reluctance of the iron $\Re_{\text {iron }}$ is negligible compared to that of air $\Re_{\text {air }}$, the latter is equivalent to a reluctance of a gap with Length e.

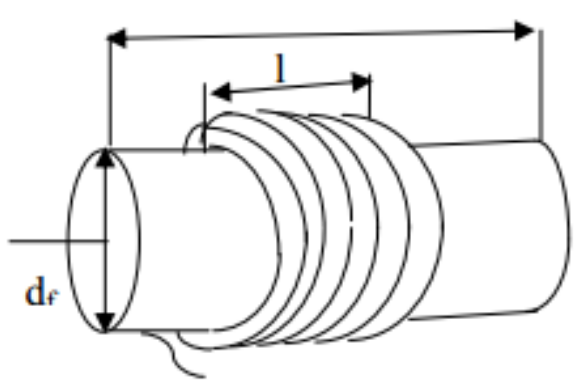

Figure 5.1. coil iron nucleus 
The section of the nucleus is given by:

$$
s_{f}=\frac{\pi d_{f}^{2}}{4}
$$

The section of the useful iron is:

$$
S_{u t i}=S_{f} k_{u t i}
$$

The induction $B$ is determined by the formula of KAPP:

$$
B=\frac{U_{e f}}{4,4 \cdot N \cdot f S_{u t i}}
$$

It can also be calculated by the formula of Hopkinson.

The length of the gap is fictional:

$$
e=0.28 \cdot \sqrt{\frac{S_{u t i} l}{l_{n}}}
$$

The volume of air to magnetize:

$$
V=e S_{u t i}
$$

The reactive power needed for the gap:

$$
Q=\frac{B^{2} \cdot \omega V}{2,5} \cdot 10^{6}
$$

The active power is low because $f$ and $B$ are weak.

The fictional absorbed current is:

$$
I_{r}=\frac{Q}{U_{e f}}
$$

This current can be considered as total current, which can also be determined by applying the formula of Hopkinson.

$$
N I_{\text {ef }}=9 \text {. Q }
$$

So:

$$
I_{e f}=\frac{\Re \cdot \phi_{m}}{\mathrm{~N} \cdot \sqrt{2}}=\frac{1}{s}\left(\frac{l_{n}}{\mu_{0} \cdot \mu_{r}}+\frac{e}{\mu_{0}}\right) \cdot \frac{\phi_{\mathrm{m}}}{\mathrm{N} \cdot \sqrt{2}}
$$

With: 


$$
\frac{l}{\mu_{0} \cdot \mu_{r}} \approx 0
$$

The impedance of the circuit is:

$$
Z=L \cdot \omega=\frac{U_{e f}}{I_{e f}}
$$

When the inductance of the coil is:

$$
L=\frac{Z}{\omega}
$$

A MATLAB program has been designed to perform calculations of inductance for a coil with centered nucleus.

\section{The Coil Characteristics}

The characteristics of the coil considered are shown in the table 6.1 , which summarizes all the electrical and geometrical dimensions.

\begin{tabular}{llcl} 
& \multicolumn{3}{c}{ Table 4.1. Electrical and geometrical dimensions of otlm } \\
\hline Elements & Dimensional & Symbols & Values \\
\hline Coil & Length & I & $22.0[\mathrm{~cm}]$ \\
& Outside diameter Inside diameter & $\mathrm{d} \mathrm{d0}$ d L0 & $6.4[\mathrm{~cm}]$ \\
& Conductor diameter Inductance & $\mathrm{L}$ & $3.0[\mathrm{~cm}$ \\
& Without cor & $\mathrm{R}$ & $1[\mathrm{~mm}]$ \\
& nucleus inductor internal resistance & & $0.04[\mathrm{H}]$ \\
& & & $1.1[\mathrm{H}]$ \\
& & & $10[\Omega]$ \\
Nucleus & Length & If & \\
& Diameter & df & $26[\mathrm{~cm}]$ \\
& Mass & $\mathrm{m}$ & $2.9[\mathrm{~cm}]$ \\
& & & $0.65[\mathrm{~mm}]$ \\
Air gap & Thickness & e1 & $0.1[\mathrm{~mm}]$ \\
\hline
\end{tabular}

\section{Calculation and Visualization of Inductors According to the Position of the Nucleus}

The results of simulation of the inductances $\mathrm{L}_{0}, \mathrm{~L}_{\mathrm{f}}, \mathrm{M}$ and $\mathrm{L}$ in function of displacement $(x)$ of the nucleus effected under MATLAB program, are giving by the following figures:

Figure 7.1 represents the variation of the nucleus position inside the coil. We note that during the penetration of iron in the coil and chasing air, the length of it increases gradually to its maximum value which corresponds to the total occupation, then a regression of the curve that corresponds to $t$ progressive removal core, knowing that the length of the coil $I=I_{0}+I_{f}$.

The curve of evolution of the air inside the coil is represented by Figure 7.1 and on which are superimposed the two curves $I_{0}$ and $I_{f}$. 


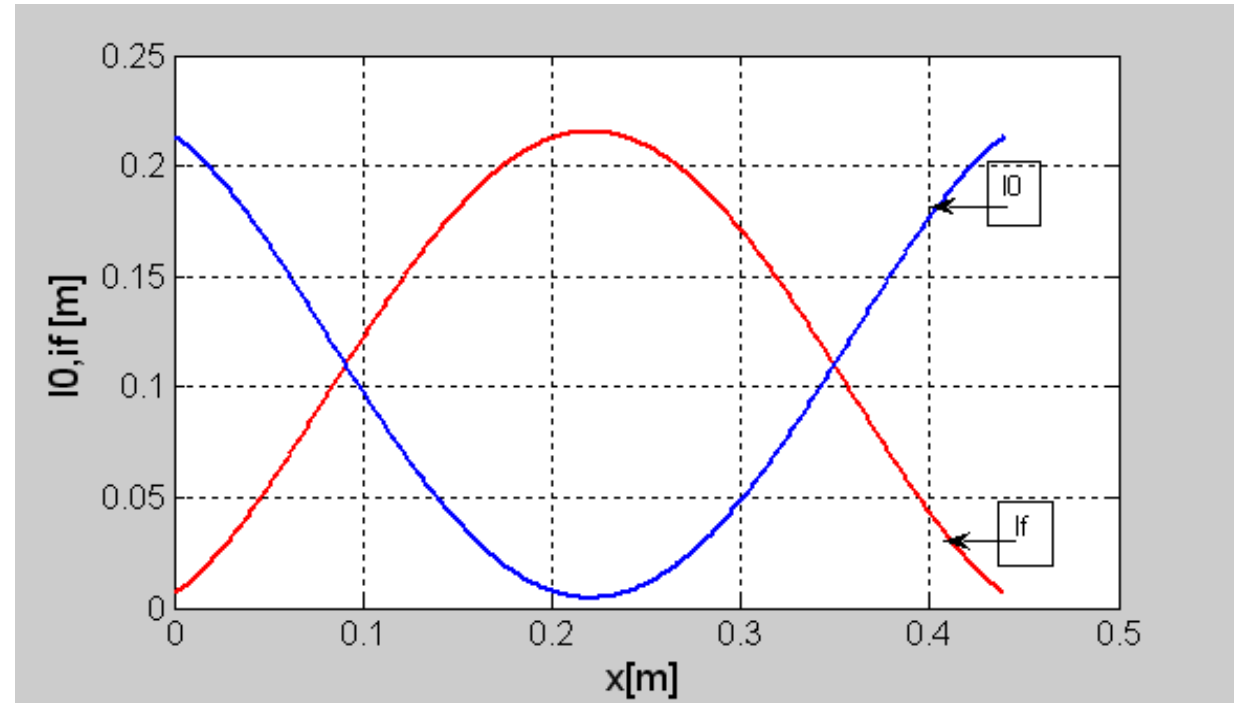

Figure 7.1. Superposition of $\mathrm{I}_{0}=\mathrm{f}(\mathrm{x})$ and $\mathrm{I}_{\mathrm{f}}=\mathrm{g}(\mathrm{x})$

Figure 7.2 represents the inductance of the coil with movement of the nucleus in function of the nucleus position inside the coil. When the nucleus is in center, $L_{0}$ pass by its minimum and it takes again its proper value when it is completely clear.

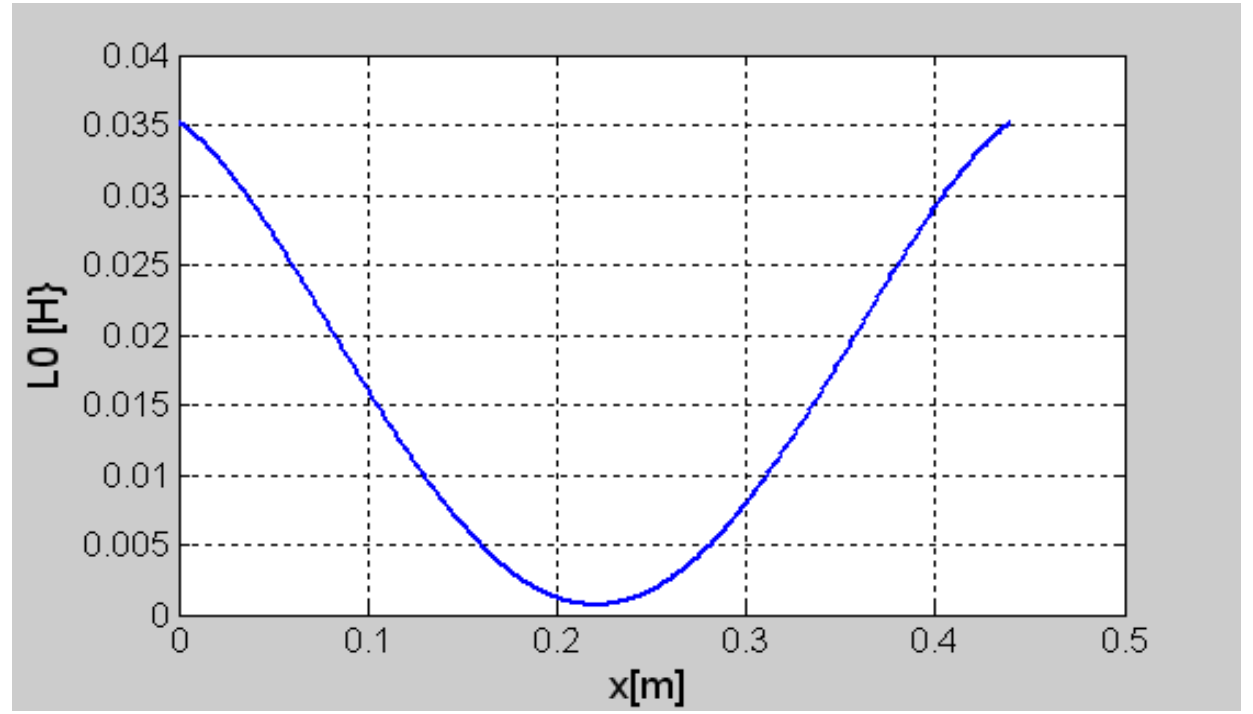

Figure 7.2. Inductance of the air in function of the nucleus position

The figure 7.3 represents the inductance of iron in function of the position of the nucleus. It takes minimum values outside of the coil and a maximum value when the nucleus is in central. 


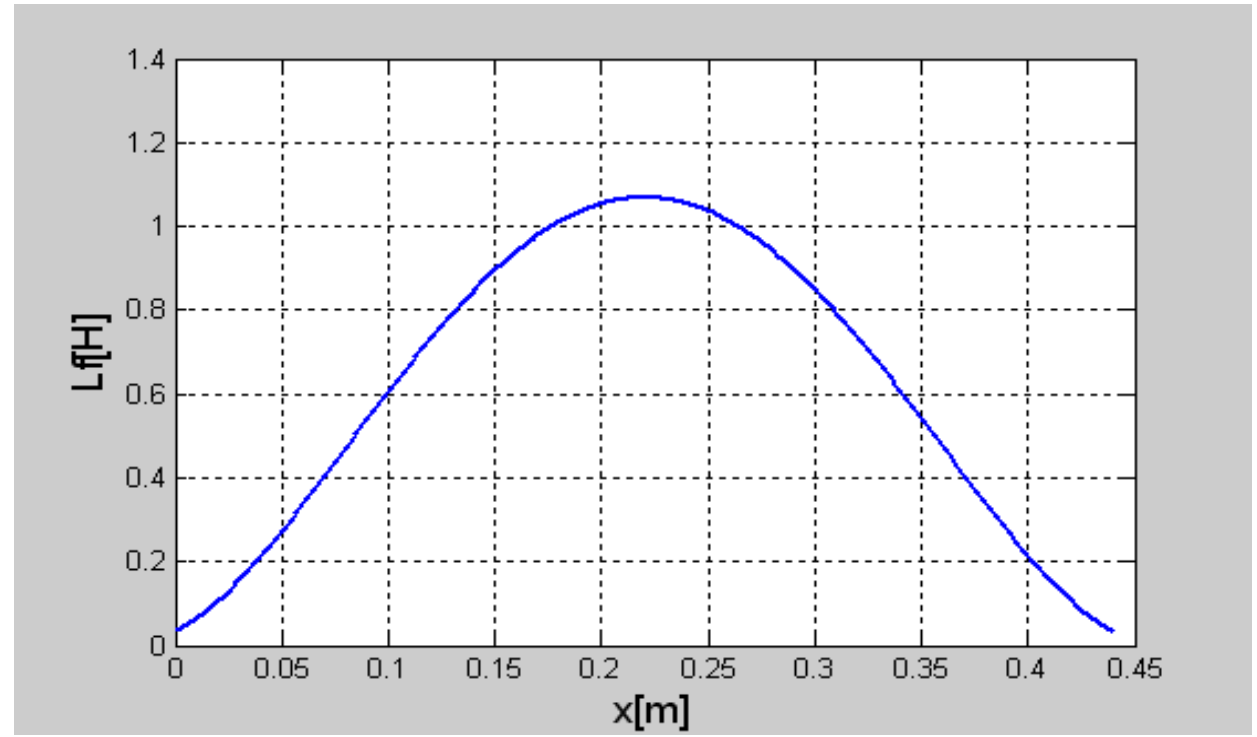

Figure 7.3. Inductance of iron in function of the position of the nucleus

Figure 7.4 shows the variation of mutual inductance in function of its position inside the coil and takes the most important values almost to this of the total inductance of the coil.

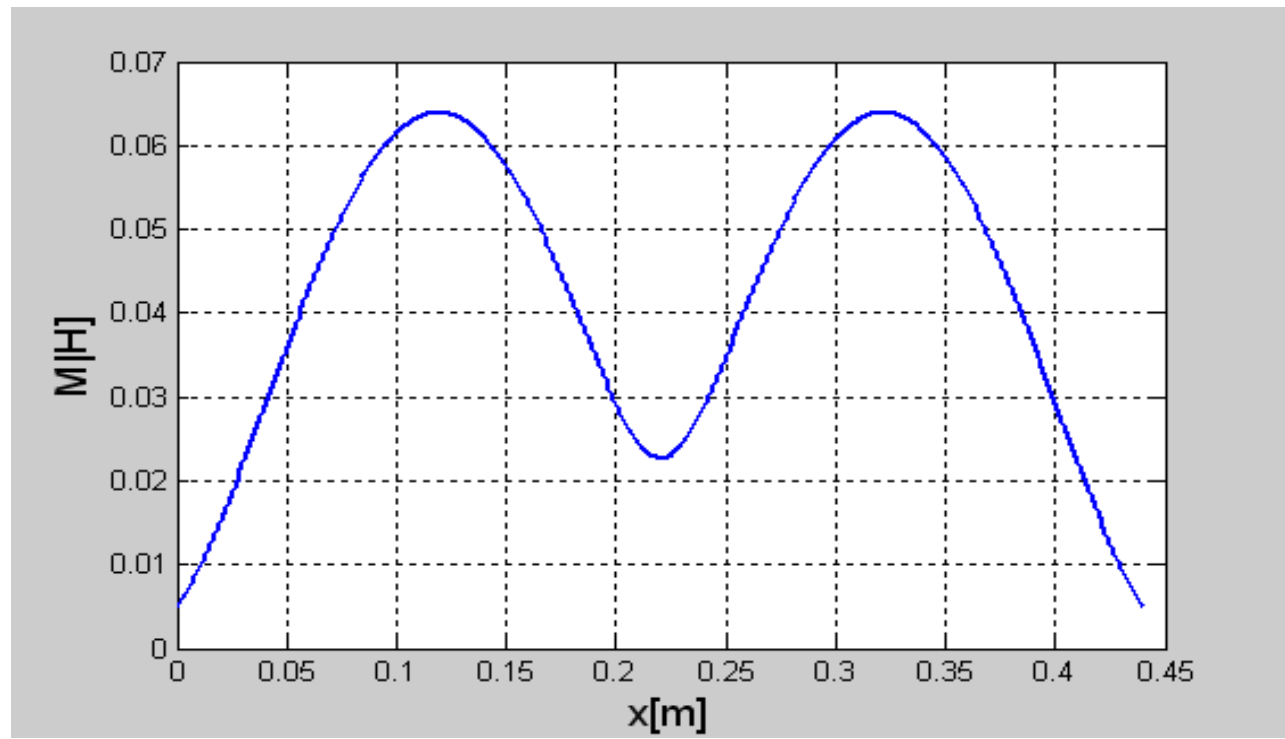

Figure 7.4. Variation of the mutual-inductance in function of $(X)$

The total inductance of the coil is represented by curve 7.5 , we notice that it is affected by the presence of the nucleus and it takes a maximum value equal to $1.1 \mathrm{Henry}$. 


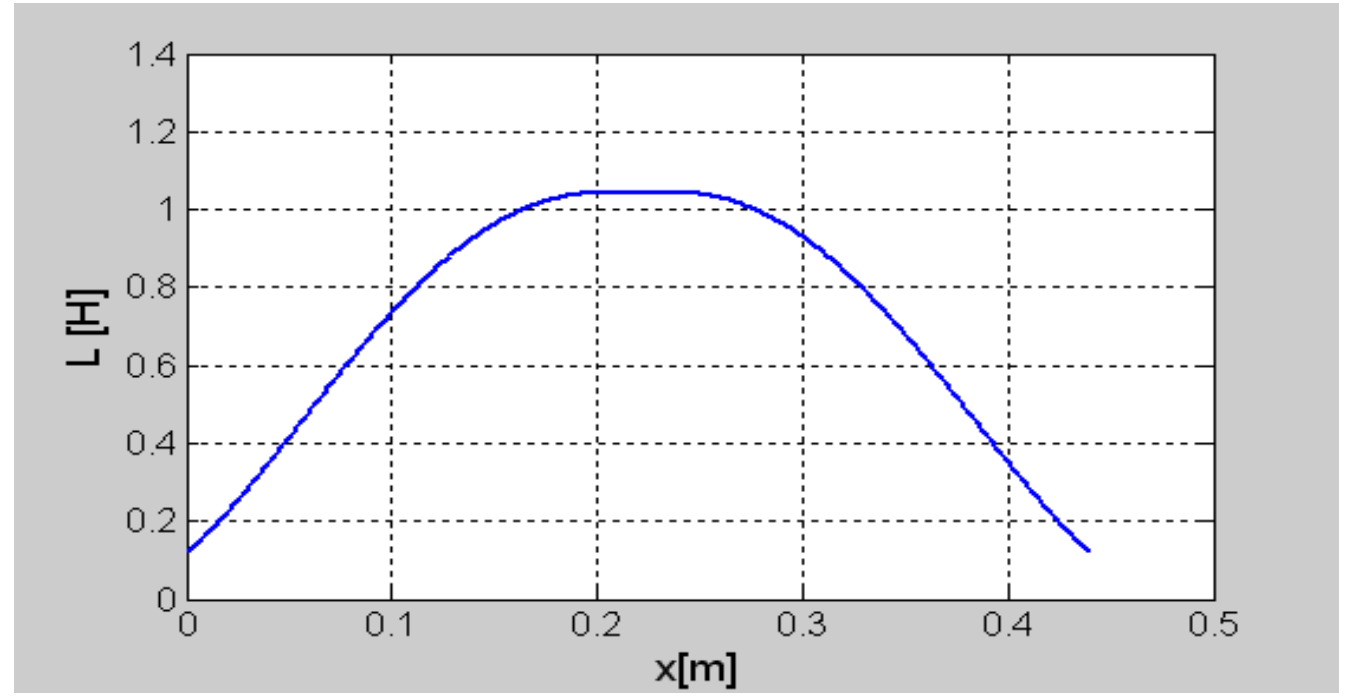

Figure 7.5. Variation of the Total Inductance in Function of $(X)$

\section{Conclusion:}

The elaborate work have give a satisfactory results, the inductances determined can be exploit for any eventual simulation of a tubular linear motor all we exploit the equations of KIRCHOFF in level of the inductor (coil) and induces (nucleus) to model the (C.I.N.C). (C.I.N.C)

The results of the simulation, presented in the paper, illustrate the performance of the

\section{References}

[1] E Levi and M Panzer. Electromechanical Power Conversion. New York: McGraw-Hill. 1966: 9.

[2] George Asch. les capteurs en Instrumentation industrielle. Dunod Edition. 1987.

[3] D HEDJAZI and A Chaghi and R Abdessamed. "Modelling and characterization of High Performance Linear Oscillating Parametric Motor". Jee Journal of Electrical Engineering. vol. 8, No 1, Poitechnica of Timisoara, Romania. ISSSN 1582-4594, 2007.

[4] S Kikuchi and K Ishikawa. "A New Type 4-Legged Linear Parametric Motor with Excellent Performance". IEEE Transactions on magnetic. 1997; 33(5).

[5] K Ishikawa and M Ishizuka and S Kikuchi. "Performance Characteristics of A New Type of Linear Parametric Motor With Double Driving Surfaces". IEEE Transactions on magnetic. 1998; 34(4).

[6] BZ Kaplan. "Analysis of a method for magnetic Levitation". Proc. Inst. Elec. Eng. 1967; 114: 18011804.

[7] BZ Kaplan. "A note on parametric machines". Proc. IEEE. 1966; 54: 898.

[8] K Ishikawa and S Kikuchi. "Improvement of the Performance characteristics of a linear parametric motor with Open Magnetic Circuit". IEEE Transactions on magnetic. 1999; 35(5). 